\title{
Efecto de la temperatura en respuestas fisiológicas de la concha de abanico Argopecten purpuratus
}

\section{Effect of temperature on physiological responses of Peruvian scallop Argopecten purpuratus}

\section{Jhon Dionicio Acedo* y Jorge Flores Mego}

*Autor para correspondencia

Laboratorio de Ecofisiología Acuática, Instituto del Mar del Perú (IMARPE), Esquina Gamarra y General Valle S/N Chucuito, Callao, Perú.

E-mail Jhon Dionicio: jdionicio@imarpe.gob.pe

E-mail Jorge Flores: jflores@imarpe.gob.pe

\section{Resumen}

Se determinó el efecto de la temperatura sobre la tasa de aclaramiento (TA), ingestión (TI) y consumo especifico de oxígeno (CEO) en individuos de Argopecten purpuratus (Lamarck, 1891) de distintos grupos de tallas. Las pruebas de TA y TI se realizaron a una concentración de Chaetoceros calcitrans de aproximadamente $1 \times 10^{6}$ cel. $\mathrm{mL}^{-1}$, se evaluaron dos temperaturas 17 y $22^{\circ} \mathrm{C}$ y se conformaron diferentes grupos de tallas promedio de $7.6 \pm 0.265,4.9 \pm 0.058$ y $3.7 \pm 0.173 \mathrm{~cm}$. En las pruebas de CEO los grupos de tallas promedio fueron $8.1 \pm$ $0.351,5.6 \pm 0.058$ y $4.3 \pm 0.100 \mathrm{~cm}$. Los resultados muestran un efecto significativo de la temperatura en la TA $\left(\right.$ L. $\left.\mathrm{h}^{-1}\right)$ y $\mathrm{Tl}\left(\right.$ cel. $\left.\mathrm{h}^{-1} \times 10^{5}\right)$ sobre los individuos de mayor tamaño $(7.6 \pm 0.265 \mathrm{~cm})$, observándose a $22^{\circ} \mathrm{C}$ un incremento promedio, respecto a $17^{\circ} \mathrm{C}$, de hasta $250 \%$ para TA y $48 \%$ para TI. Además, se observó una relación directa del tamaño corporal con las TA y TI en ambas temperaturas. Se registró el efecto de la temperatura de $22^{\circ} \mathrm{C}$ sobre CEO en todos los grupos evaluados, con un incremento de $239.8,165.3$ y $183.4 \%$ para individuos de tallas de $8.1 \pm 0.351,5.6 \pm 0.058$ y $4.3 \pm 0.100$ respectivamente. Asimismo, los resultados muestran una relación indirecta del tamaño corporal con la CEO, en ambas temperaturas evaluadas.

Palabras clave: concha abanico; bioenergética; tasa de aclaramiento; sistema de recirculación; tasa fisiológica.

\section{Abstract}

The effect of temperature on the clearance rate (CR), ingestion rate (IR) and specific oxygen consumption (SOC) in individuals of Argopecten purpuratus at different size groups were determined. The CR and IR tests were performed at a concentration of approximately $1 \times 10^{6} \mathrm{cel} . \mathrm{mlL}^{-1}$ of Chaetoceros calcitrans, two temperatures 17 and $22^{\circ} \mathrm{C}$ were evaluated and different groups of average size were formed of $7.6 \pm 0.265$, and $0.058 \pm 4.9$ $3.7 \pm 0.173 \mathrm{~cm}$. In SOC test the average size groups were $8.1 \pm 0.351,0.058 \pm 5.6$ and $4.3 \pm 0.100 \mathrm{~cm}$. The results show a significant effect of temperature in $\mathrm{CR}\left(\mathrm{Lh}^{-1}\right)$ and IR $\left(\right.$ cel. $\left.\mathrm{h}^{-1} \times 10^{5}\right)$ on the larger individuals $(7.6$ $\pm 0.265 \mathrm{~cm}$ ), it was observed at $22^{\circ} \mathrm{C}$ an average increase, about $17{ }^{\circ} \mathrm{C}$, up to $250 \%$ to $\mathrm{CR}$ and $48 \%$ to IR. In addition, a direct relationship of body size with $\mathrm{CR}$ and IR in both temperatures was observed. The effect of temperature at $22^{\circ} \mathrm{C}$ on SOC in all groups was evaluated, with an increase of $239.8,165.3$ and $183.4 \%$ for size individuals of $8.1 \pm 0.351,0.058 \pm 5.6$ and $4.3 \pm 0.100$ respectively. Furthermore, in both evaluated temperatures, the results show an indirect relationship of body size with the SOC.

Keywords: Peruvian scallop; bioenergetics; clearance rate; recirculation system; physiological rate.

\section{Citación:}

Dionicio Acedo J. \& J. Flores Mego. 2015. Efecto de la temperatura en respuestas fisiológicas de la concha de abanico Argopecten purpuratus. Revista peruana de biología 22(3): 329 - 334 (Diciembre 2015). doi: http:// dx.doi.org/10.15381/rpb.v22i3.11439
Información sobre los autores:

JD y JF, realizaron el diseño experimental, los experimentos y análisis de datos. JD redactó el manuscrito; JF revisó y aprobó el manuscrito. Los autores no incurren en conflictos de intereses.
Presentado: $\quad 31 / 07 / 2015$

Aceptado: $\quad 13 / 11 / 2015$

Publicado online: 18/12/2015
Fuente de financiamiento:

Recursos ordinarios del Instituto del Mar del Perú - IMARPE. 


\section{Introducción}

La concha de abanico Argopecten purpuratus (Lamarck 1819) es una de las especies de bivalvos de mayor importancia en Perú debido a su gran demanda en el mercado nacional e internacional, principalmente en Francia (PRODUCE 2012); lo que ha llevado a un sostenido incremento en su producción. Luego del "boom" de concha de abanico por efecto de El Nińo 1997/98 (Mendo \& Wolff 2003), las condiciones de agua templadas en la Bahía de Sechura (Piura) originaron un incremento de las biomasas de este recurso en los bancos naturales de la Isla Lobos de Tierra (Lambayeque) y de la misma bahía, convirtiéndose en uno de los bancos más importantes del Perú (Mendo et al. 2008), y que en la actualidad sustenta las exportaciones de concha abanico en el Perú, cuya producción se basa principalmente en el cultivo de fondo.

Los volúmenes de desembarques de concha abanico han incrementado considerablemente durante fuertes eventos El Nińo, tal y como sucedió en los ańos 1982/83 o 1997/98 (Mendo \& Wolff 2003), bajo estas condiciones de aguas cálidas la concha de abanico recolonizó rápidamente, incrementando su población (Wolff 1985a 1987b), al respecto Wolff y Wolff (1983), Mendo et al. (1988); Yamashiro y Mendo (1988); Mendo y Jurado (1993); coinciden al señalar que el incremento se debe a un aumento en la tasa de crecimiento.

En ecosistemas marinos someros, los cultivos de bivalvos pueden provocar un significativo impacto en los nutrientes así como del ciclo de energía, por otro lado, el incremento del metabolismo microbiano produce una reducción de las concentraciones de oxígeno disuelto. No obstante, durante periodos cálidos como el evento El Niño se observan elevadas concentraciones de oxígeno, generando con ello incrementos en las densidades de $A$. purpuratus (Wolff 1985), sin embargo, durante el evento El Niño 97/98 la producción primaria decreció significativamente (Mendo \& Wolff 2003) pero sin afectar la producción de concha abanico.

El cultivo de $A$. purpuratus se ha convertido en una actividad de importancia económica, principalmente la fase de engorde, la cual implica aprovechar las condiciones naturales del mar tales como la temperatura, oxígeno disuelto, oferta alimentaria, nutrientes, entre otros. Sin embargo, esta actividad se ha desarrollado de manera empírica, desconociéndose las relaciones entre las condiciones ambientales y las respuestas fisiológicas de $A$. purpuratus, conocimiento que permitiría tomar decisiones para mantener o mejorar la productividad de este recurso.

En este sentido, se vienen realizando esfuerzos orientados a estimar la capacidad de carga en el cultivo de $A$. purpuratus, definida como la máxima población o biomasa que un área puede soportar de manera sostenible. Al respecto, tasas fisiológicas como las tasas de filtración (TF) e ingestión (TI) (Rajesh et al. 2001) y consumo específico de oxigeno (CEO) (Tam et al. 2001), permitirían estimar la capacidad de carga de los sistemas naturales utilizados para producción acuícola así como también la construcción de modelos tróficos.

Bajo el enfoque fisiológico, los organismos acuáticos varían sus tasas fisiológicas respecto a la temperatura. Por ello, el objetivo del presente trabajo fue determinar el efecto de las temperaturas $\left(17\right.$ y $\left.22^{\circ} \mathrm{C}\right)$ en las tasas de aclaramiento (TA), ingestión (TI) y tasa de consumo específico de oxígeno (CEO) en individuos de diferentes tamaños de Argopecten purpuratus en condiciones de laboratorio.

\section{Material y métodos}

Acondicionamiento en laboratorio.- Los ejemplares fueron colectados en la bahía de Sechura-Piura, en el mes de febrero del 2012 y transportados al Laboratorio de Ecofisiología Acuática en la sede central del Instituto del Mar del Perú (IMARPE), donde se distribuyeron en tres grupos de tamańo I (7.6 \pm 0.265 $\mathrm{cm})$, II $(4.9 \pm 0.058 \mathrm{~cm})$ y III $(3.7 \pm 0.173 \mathrm{~cm}) 17$ y $22^{\circ} \mathrm{C}$ para su acondicionamiento (Tabla 1), el cual se llevó a cabo en un sistema de 3 bandejas suspendidas dentro de un tanque circular de $2 \mathrm{~m}^{3}$ conectado a un sistema de recirculación con control de temperatura y aireación constante.

El periodo de acondicionamiento duró aproximadamente 20 días, con fotoperiodo 10:14 (luz: oscuridad) y alimentación con dos especies de microalgas: Chaetoceros calcitrans e Isochrysis galbana a una relación de 1:1.

Tasa de Aclaramiento e Ingestión (TA y TI).- La metodología fue basada por el Clearance Method (Método de Aclaramiento), la cual consistió en el estudio de la reducción de partículas en un tiempo determinado dentro de un sistema cerrado, donde se situó al organismo filtrador en unidades experimentales (Filgueira 2007). Para ello se utilizaron recipientes cilíndricos de 1.9 L de capacidad, agua de mar filtrada a 10, 5 y $1 \mu \mathrm{m}$, esterilizada con UV y aireación constante en cada recipiente para mantener homogénea la concentración microalgal, además se controló la temperatura durante el desarrollo del experimento.

Para el experimento se preparó una concentración de $1 \times 10^{6}$ cel. $\mathrm{mL}^{-1}$ de Chaetoceros calcitrans, además los individuos utilizados estuvieron en ayuno por $24 \mathrm{~h}$ con el fin de vaciar su contenido estomacal. Las pruebas fueron realizadas por triplicado para cada grupo, con un grupo control (recipiente sin individuo), se tomaron muestras de los recipientes a cada hora, haciendo conteos microalgales utilizando una cámara Neubauer $(0.1 \mathrm{~mm}$ de profundidad) y un microscopio compuesto.

Determinación de la Tasa de Aclaramiento (TA).- Según la metodología descrita por Conover y Huntley (1980), la tasa de Aclaramiento (TA) se estimó con la siguiente fórmula:

$\mathrm{TA}=V^{*} g / N$. (Ecuación 1)

Donde, $\mathrm{TA}=$ tasa de aclaramiento $\left(\mathrm{L} \cdot \mathrm{h}^{-1}\right.$.individuo $\left.{ }^{-1}\right), N=$ número de individuos, $V=$ volumen del recipiente $(\mathrm{mL})$ y $g=$ constante de filtración $\left(\mathrm{L} \cdot \mathrm{h}^{-1}\right)$. La constante de filtración puede ser calculada por:

$$
g=k-\left\{\left[\ln \left(C_{2} / C_{1}\right)\right] /\left(t_{2}-t_{1}\right)\right\} \ldots \ldots \ldots \ldots \text {....(Ecuación 2) }
$$

Tabla 1. Talla de individuos de Argopecten purpuratus "concha de abanico" para las pruebas de Tasa de Aclaramiento (TA) e Ingestión ( $\mathrm{TI})$, a dos temperaturas $\left(17\right.$ y $\left.22^{\circ} \mathrm{C}\right)$.

\begin{tabular}{cccc}
\hline Grupo & $\begin{array}{c}\text { Rango } \\
(\mathbf{c m})\end{array}$ & $\begin{array}{c}\text { Longitud } \\
\text { promedio } \\
\mathbf{( c m})\end{array}$ & D.E. \\
\hline I & $(7.9-7.5)$ & 7.6 & 0.265 \\
II & $(5.0-4.9)$ & 4.9 & 0.058 \\
III & $(3.6-3.9)$ & 3.7 & 0.173 \\
\hline
\end{tabular}


Donde, $k=$ crecimiento de fitoplancton (células. $\mathrm{mL}^{-1}$ ), $C_{1} \mathrm{y}$ $C_{2}$ son la concentración inicial y final (células. $\mathrm{mL}^{-1}$ ), respectivamente, en los compartimientos experimentales, $t_{1}$ y $t_{2}$ son los tiempos iniciales y finales (horas).

El crecimiento del fitoplancton $(k)$ se obtuvo al aplicar la siguiente ecuación.

$k=\left[\ln \left(C_{C T-2} / C_{1}\right)\right] /\left(t_{2}-t_{1}\right)$ (Ecuación 3)

Donde $C_{C T-2}$ es la concentración final en los compartimientos controles.

Las ecuaciones (1), (2) y (3) se aplicaron para estimar la TA para los 3 grupos y sus respectivas concentraciones.

Determinación de la Tasa de Ingestión (TI).- La tasa de ingestión (TI), de acuerdo a Conover y Huntley (1980), puede ser estimada como:

$$
I=\mathrm{TA}^{*} C_{m}
$$

(Ecuación 4)

Donde, $I=$ Tasa de ingestión ${ }^{*} 10^{5}\left(\right.$ células. $^{-1} \cdot$ ind $\left.^{-1}\right)$, TA $=$ definido anteriormente, y $C_{m}=$ concentración media de fitoplancton durante el periodo experimental. De acuerdo a McClatchie y Lewis (1986), $C_{m}$ no representa la media aritmética entre $C_{1}$ y $C_{2}$ sino que, dado que el crecimiento del fitoplancton tiende a ser logarítmico, una estimación adecuada de $C_{m}$ debería de ser la media geométrica. Es así que la ecuación sugerida es:

$$
C_{m}=\left[C_{1}[\exp ((k-g) t)-1] /[t(k-g)] \ldots \ldots\right. \text { (Ecuación 5) }
$$

Donde $C_{m}=$ Concentración media estimada (células. $\mathrm{mL}^{-1}$ ), $t_{2}-t_{1}$ y $C, k$ y $g$ se definieron anteriormente.

Tasa de Consumo Específico de Oxígeno (CEO).- Se evaluaron nueve individuos, tres por cada grupo (Tabla 2), bajo el concepto de sistema estático, donde se colocó un individuo por respirómetro, además se contó con un grupo control (respirómetro sin individuo). Los individuos utilizados estuvieron en ayuno por $24 \mathrm{~h}$ con el fin de evitar la influencia del proceso de digestión en la respuesta metabólica.

Los cálculos de CEO se realizaron utilizando la fórmula.

$$
\begin{aligned}
& C M O=\left(O_{t}-O_{o}\right) *\left(V_{c}-V_{m}\right) / t \\
& \mathrm{CEO}=C M O / P
\end{aligned}
$$

Donde, CMO: consumo medio de oxígeno $\left(\mathrm{mg} \mathrm{O}_{2} \cdot \mathrm{h}^{-1}\right.$.ind $\left.{ }^{1}\right)$; CEO: consumo específico de oxígeno $\left(\mathrm{mg} \mathrm{O}_{2} \cdot \mathrm{h}^{-1} \cdot \mathrm{g}^{-1}\right) ; O_{t}$ : concentración de oxígeno en un tiempo t; $O_{0}$ : concentración de oxígeno al inicio; $V_{c}:$ volumen de cámara respirométrica $(\mathrm{L}) ; V_{o}$ : volumen del organismo (L); $t$. periodo de tiempo (h); $P$ : peso seco del organismo (g). El peso seco de las muestras fue determinado en una estufa a $70{ }^{\circ} \mathrm{C}$ por $48 \mathrm{~h}$.

Tabla 2. Talla de individuos de Argopecten purpuratus, "concha de abanico" para las pruebas de CEO a 17 y $22{ }^{\circ} \mathrm{C}$.

\begin{tabular}{cccc}
\hline Grupo & $\begin{array}{c}\text { Rango } \\
(\mathbf{c m})\end{array}$ & $\begin{array}{c}\text { Longitud } \\
\text { promedio } \\
\mathbf{( c m )}\end{array}$ & D.E. \\
\hline I & $(7.7-8.4)$ & 8.1 & 0.351 \\
II & $(5.6-5.7)$ & 5.6 & 0.058 \\
III & $(4.2-4.4)$ & 4.3 & 0.100 \\
\hline
\end{tabular}

Para las evaluaciones de los datos se ajustaron modelos exponenciales en función de la temperatura a las distintas tasas fisiológicas; TA, TI y CEO.

\section{Resultados}

Tasa de Aclaramiento (TA).- Los resultados muestran un efecto significativo de la temperatura en la TA sobre los individuos de mayor tamaño $(7.6 \pm 0.265 \mathrm{~cm})$, observándose a $22^{\circ} \mathrm{C}$ un incremento promedio en la TA de hasta 2.5 veces respecto a $17^{\circ} \mathrm{C}$, sin embargo en individuos más pequeños $(3.7 \pm 0.173$ $\mathrm{cm}$ ) la temperatura no mostró influencia en las TA (Fig. 1).

Los mayores valores de TA se muestran en los individuos de tamaño promedio de $7.6 \mathrm{~cm}$, con un valor máximo de 4.86 L. $\mathrm{h}^{-1}$, observándose diferencias significativas $(\mathrm{p}<0.05)$ con la TA de los ejemplares de longitud promedio de $3.7 \mathrm{~cm}$, donde se obtuvieron tasas de 0.24 L.h $^{-1}$ (Fig. 1).

Tasa de Ingestión (TI).- La respuesta de TI se vio afectada fuertemente por la temperatura en los ejemplares $7.6 \pm 0.265$ cm y $3.7 \mathrm{~cm} \pm 0.173 \mathrm{~cm}$, observándose a $22{ }^{\circ} \mathrm{C}$ incrementos del 48 y 22\%, respectivamente (Fig. 2), sin embargo, en los individuos de $4.9 \pm 0.173 \mathrm{~cm}$, se observa una independencia de la TI en ambas temperaturas.

Los resultados muestran una relación directa entre la TI y el tamaño corporal, observándose diferencias significativas $(\mathrm{p}<0.05)$ entre todos los grupos, con un valor máximo de $9.88 \times 10^{5}$ cel. $\mathrm{h}^{-1}$ en los individuos de $7.6 \pm 0.265 \mathrm{~cm}$ de longitud promedio.

Tasa de Consumo Específico de Oxígeno (CEO). - La CEO mostró dependencia con la temperatura, observándose a $22{ }^{\circ} \mathrm{C}$ las mayores tasas, con valores máximos de 0.64062, 1.42052 y $8.04801 \mathrm{mg} \mathrm{O} \cdot \mathrm{h}^{-1} \cdot \mathrm{g}^{-1}$ respectivamente para los individuos de los Grupos I, II y III (Fig. 3)

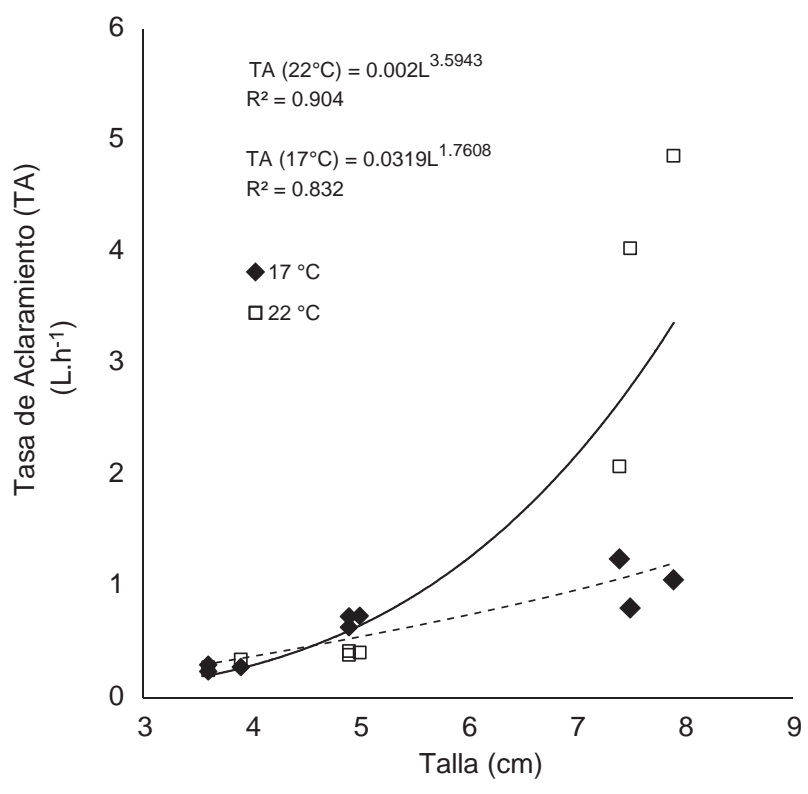

Figura 1. Tasa de Aclaramiento $\left(T A-L . h^{-1}\right)$ en individuos de concha de abanico a la concentración microalgal de $1 \times 10^{6}$ cel. $\mathrm{mL}^{-1}$ acondicionados a 17 y $22^{\circ} \mathrm{C}$. 


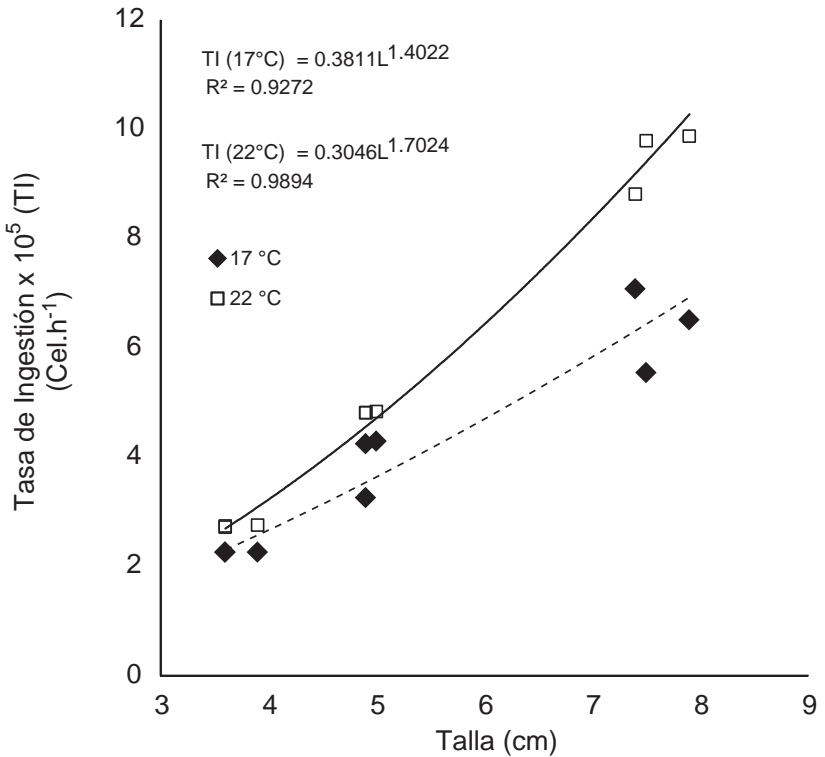

Figura 2. Tasa de Ingestión x $10^{5}\left(\mathrm{TI}-\right.$ cel. $\left.^{-1}\right)$ en diferentes tamaños de individuos de concha de abanico a la concentración microalgal de $1 \times 10^{6} \mathrm{cel} . \mathrm{mL}^{-1}$ acondicionados a 17 y $22^{\circ} \mathrm{C}$.

La CEO varió de manera inversa al tamaño corporal en ambas temperaturas, observándose a $22^{\circ} \mathrm{C}$ diferencias significativas entre los grupos evaluados, sin embargo a $17^{\circ} \mathrm{C}$ no se encontraron diferencias entre las CEO de los grupos I y II.

\section{Discusión}

El crecimiento de los organismos bajo condiciones naturales es la resultante de la interacción de factores ambientales (corriente, oxígeno disuelto, temperatura), biológicos (alimento, variables fisiológicas y reproducción del organismo) y tecnológicos (sistemas de cultivos que tiene diferentes valores de

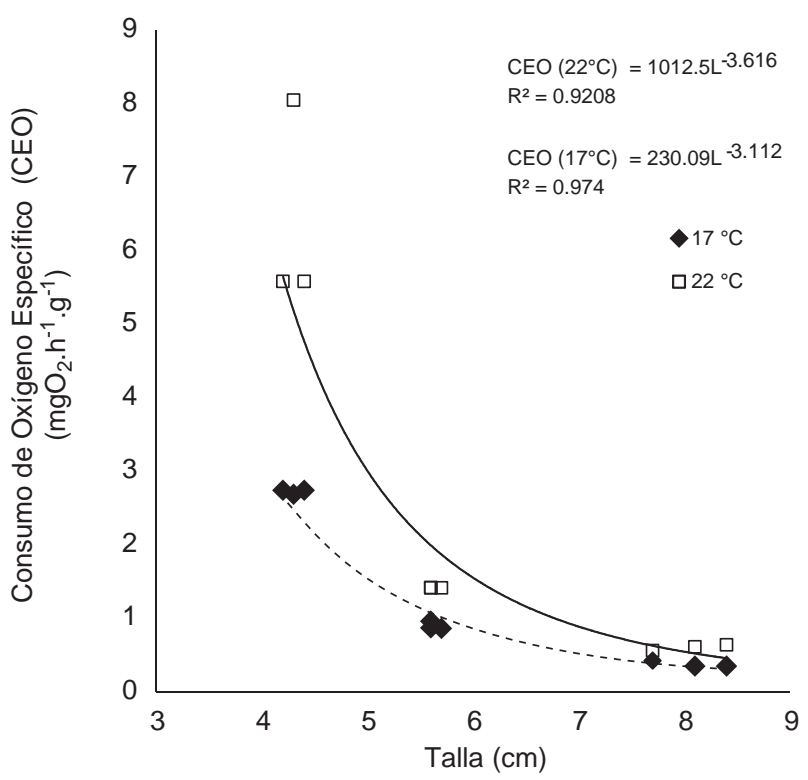

Figura 3. Tasa de Consumo Específico de Oxígeno (CEO $\mathrm{mgO} 2 . \mathrm{h}^{-1} \cdot \mathrm{g}^{-1}$ ) en diferentes tamaños de Argopecten purpuratus "concha de abanico" cultivados a $17^{\circ} \mathrm{C}$ y $22^{\circ} \mathrm{C}$. eficiencia), sin embargo en la mayoría de estudios ecofisiológicos en bivalvos han evaluado individualmente las variables. $\mathrm{Al}$ respecto, un ascenso de la temperatura incrementa la mayoría de los procesos fisiológicos de bivalvos, tales como la tasa de filtración (TF) e ingestión (TI), por tanto, en ambiente natural la concha de abanico incrementa su crecimiento y reproducción ante un evento El Niño debido a la entrada de aguas cálidas, sin embargo el costo metabólico a estas condiciones requiere una fuente de alimento; tanto en variedad, cantidad y calidad para sostener el crecimiento y desarrollo de su población (Mendo \& Wolff 2003). Bajo este supuesto se ha estudiado el efecto de la temperatura en las tasas de filtración (Schulte 1975, Ian 2004, Mona et al. 2011, Nieves et al. 2013) e ingestión (Mona et al. 2011) en bivalvos marinos obteniendo como resultado común una relación directa, tanto de la TF y TI con la temperatura.

En este contexto, el presente trabajo muestra un aumento significativo de las tasas fisiológicas en los individuos de mayor tamaño $(7.6 \pm 0.256 \mathrm{~cm})$ a $22^{\circ} \mathrm{C}$, de hasta 2.5 y 1.5 veces para la TA y TI, respectivamente; debiéndose principalmente al incremento metabólico por efecto de la temperatura en ambos casos, estos resultados presentaron el mismo patrón con los reportes obtenidos por Ian (2000), quien determinó en Pecten maximus la mayor tasa de consumo de alimento a $23{ }^{\circ} \mathrm{C}$, asimismo Ian (2004) trabajando con la misma especie reportó un efecto significativo en la TF a $21^{\circ} \mathrm{C}$ respecto a $16^{\circ} \mathrm{C}$.

En los individuos de menor tamaño, la temperatura no mostró un efecto tanto en la TA como en la TI, lo cual podría ser originado a la alta concentración microalgal de Ch. calcitrans $\left(1 \times 10^{6}\right.$ cel. $\left.\mathrm{mL}^{-1}\right)$, interpretándose como un umbral de saturación para individuos de este tamańo, originando el cese o disminución de su TA y TI, en este sentido, este comportamiento podría ser explicado como un ajuste fisiológico para la optimización energética y no sólo como una simple respuesta a la exposición en condiciones adversas (Winter 1978, Bayne et al. 1993, Velasco \& Navarro 2002, Filgueira et al. 2009), asimismo en el proceso podrían estar involucrados la selección y el rechazo de alimento a nivel del ctenidio y los palpos labiales (Shumway et al. 1985, Bayne et al. 1993, Ward \& Shumway 2004).

Estudios sobre el efecto del tamaño corporal sobre la TF (Navarro \& Winter 1982, Filgueira 2007) y TI (Navarro \& Winter 1982, Rajesh et al. 2001), han mostrado patrones similares con los obtenidos en nuestro estudio, por lo tanto, las mayores TA y TI mostraron dependencia por las tallas de $A$. purpuratus, posiblemente a un alto desarrollo del área branquial, permitiéndole a los animales aumentar la capacidad de filtración y posteriormente la ingesta del alimento.

Algunos autores han demostrado cómo el aumento de la temperatura incrementa la tasa de consumo de oxígeno en bivalvos (Gunasingh et al. 2002), por ello, el consumo de oxigeno es un indicador del metabolismo de los organismos y permite medir la condición fisiológica de éstos (Rodhouse 1978). En el presente estudio, los valores de la tasa de consumo específico de oxígeno (CEO) en todos los grupos de $A$. purpuratus se vio fuertemente influenciada por la temperatura, coincidiendo con los reportes de Bricelj et al. (1987) quienes trabajaron con Argopecten irradians irradians, de acuerdo a ello, se ha determinado un aumento de la tasa metabólica debido al incremento de la temperatura. Al respecto, una de las razones por la cual se originó el "boom" del stock de concha abanico en la Bahía Independencia durante 
el evento El Niño 82-83 se debería al incremento del oxígeno disuelto en el mar (Mendo \& Wolff 2003) y debido a ello, el efecto combinado de la temperatura, disponibilidad de oxígeno y el alimento coadyudan a cubrir el costo energético de la especie durante la temporada de aguas cálidas.

En el presente estudio, en ambas temperaturas, se determinó la dependencia de la CEO con el peso corporal, mostrando una relación inversa entre estas variables, en este sentido las mayores demandas de energía metabólica se observaron en organismos de menor tamańo, por lo cual los resultados evidencian una influencia directa del estado fisiológico con el nivel metabólico.

En nuestro trabajo se aprecia el efecto significativo de la temperatura sobre los individuos de mayor tamaño, este comportamiento se estaría dando debido a un alto costo energético por el mantenimiento, así como la maduración de gametos y desove (Rodhouse 1978), al respecto Deslous-Paoli y Héral (1988), mencionaron la pérdida del 18\% de energía para el esfuerzo reproductivo en el primer ańo de ostras y alrededor del $63 \%$ durante el segundo año. De acuerdo a ello, los requerimientos nutricionales, tanto en variedad, cantidad y calidad del alimento deberán compensar los costos energéticos, por ello las siguientes investigaciones deberían enfocarse en estudios orientados a modelos de capacidad de carga, considerando principalmente las variables de temperatura, alimento y oxígeno disuelto, es decir, desarrollando simulaciones de las diferentes estaciones anuales, en las diferentes zonas de cultivos y bancos naturales. Asimismo, con ayuda de los modelos predecir comportamientos frente a la presencia de eventos anómalos, tal como el Fenómeno "El Niño". Por ello estos trabajos deberían de ser un tema de atención de los usuarios de las Bahías y en especial de aquellos cuya actividad principal es el repoblamiento de concha de abanico en la Bahía de Sechura con la finalidad de minimizar los riesgos de sobrecarga del sistema que podría colapsar esta actividad.

\section{Agradecimientos}

Los autores agradecen al Ing. M.Sc. Arturo Aguirre por sus opiniones y comentarios en el presente trabajo.

\section{Literatura citada}

Bayne B.L., J.I.P. Iglesias, A.J.S Hawkins, E. Navarro, M. Heral \& J.M. Deslous-Paoli. 1993. Feeding behaviour of the mussel Mytilus edulis: Responses to variations in quantity and organic content of the seston. Journal of the Marine Biological Association of the United Kingdom, 73(04), 813-829. http://dx.doi.org/10.1017/McCS0025315400034743.

Bricelj, V. M., Epp, J., \& Malouf, R. E. (1987). Comparative physiology of young and old cohorts of bay scallop Argopecten irradians irradians (Lamarck): mortality, growth, and oxygen consumption. Journal of Experimental Marine Biology and Ecology, 112(2), 73-91. http://dx.doi.org/10.1016/00220981(87)90110-9

Conover R. \& M. Huntley. 1980. General Rules of Grazing in pelagic ecosystems. In: Primary productivity in the sea. Falkowsky (Ed.) Plenum Press, New York. Pp. 461-485. http://dx.doi. org/10.1007/978-1-4684-3890-1_25

Deslous-Paoli J.M. \& M. Héral. 1988. Biochemical composition and energy value of Crassostrea gigas (Thunberg) cultured in the bay of Marennes-Oléron. Aquatic Living Resource 1:239249. http://dx.doi.org/10.1051/alr:1988024

Filgueira R., M.J. Fernández \& U. Labarta. 2009. Tasa de Aclaramiento del mejillón Mytilus galloprovincialis. I. Respuesta a intervalos extremos de clorofila. Ciencias Marinas, 35(4):405417. <http://www.scielo.org.mx/scielo. php?pid=S018538802009000400007\&script=sci_arttext>
Filgueira R. 2007. Comportamiento alimentario del mejillón Mytilus galloprovincialis (Lamarck, 1819) en la condiciones tróficas de las Rías Gallegas. Tesis para optar el grado de Doctor en Ciencias Marinas. Instituto de Investigaciones Marinas (I.I.M. - C.S.I.C.). Pp. 135. http://digital.csic.es/ handle/10261/4261.

Gunasingh J., K. Nandakumar, K. Jesudoss, J. Azariah, K. Satapathy, $\&$ K. Nair. 2002. Influence on the temperature on the physiological responses of the bivalve Brachidontes striatulus and its significance in fouling control. Marine Environmental Research. 53: 51-63. http://dx.doi.org/10.1016/S01411136(01)00109-X.

Ian L. 2000. Effect of temperature and rate growth and condition of king scallop (Pecten maximus) spat. Aquaculture 183: 325334. http://dx.doi.org/10.1016/S0044-8486(99)00262-8

Ian L. 2004. Filtration of king scallops (Pecten maximus). Aquaculture 240: 369-384. http://dx.doi.org/10.1016/j.aquaculture.2004. 02.002

McClatchie S. \& M. Lewis. 1986. Limitation of grazing rate equations: The case for time-series measurements. Marine Biology 92:135-140. doi: 10.1007/BF00392755

Mendo J. \& E. Jurado. 1993. Length-based growth parameter estimates of the Peruvian scallop (Argopecten purpuratus). Fisheries Research. 15: 357-367. http://dx.doi.org/10.1016/01657836(93)90086-M

Mendo J., V. Valdivieso \& C. Yamashiro. 1988. Cambios en densidad, número y biomasa de la concha de abanico (Argopecten purpuratus) en la Bahía Independencia (Pisco, Perú) durante 1984 - 1987. Recursos y dinámica del ecosistema de afloramiento Peruano. Boletín Instituto del Mar del Perú-Callao, Volumen extraordinario. 163-168. < http://biblioimarpe. imarpe.gob.pe:8080/handle/123456789/1096> (Acceso $24 / 01 / 15)$

Mendo J. \& M. Wolff. 2003. El impacto de El Niño sobre la producción de concha abanico (Argopecten purpuratus) en Bahía Independencia, Pisco, Perú. Ecología Aplicada, 2(1), 7p. http://www.scielo.org.pe/scielo.php?pid=S1726-221620 03000100008\&script=sci_arttext

Mendo J., Wolff, M., Carbajal, W., Gonzáles, I. y Badjeck, M. 2008. Manejo y explotación de los principales bancos naturales de concha de abanico (Argopecten purpuratus) en la costa Peruana. En A. Lovatelli, A. Farías e I. Uriarte (eds). Estado actual del cultivo y manejo de moluscos bivalvos y su proyección futura: factores que afectan su sustentabilidad en América Latina. Taller Técnico Regional de la FAO. 20-24 de agosto de 2007, Puerto Montt, Chile. FAO Actas de Pesca y Acuicultura. No. 12. Roma, FAO. pp. 101-114.

Mona M.H., G.M. Elkhodary \& A.M. Khalil. 2011. Combined effects of temperature and algal concentration on filtration and ingestión rates of Crassostrea gigas: Bivalvia. Life Science Journal. 8(4): 805-813. http://www.lifesciencesite.com/. (Acceso 2/12/14)

Navarro J.M. \& J.E. Winter. 1982. Ingestion Rate, assimilation efficiency and energy balance in Mytilus chilensis in relation to body size and different algal concentrations. Marine Biology. 67: 255-266. http://dx.doi.org/10.1007/BF00397666

Nieves M., L. Buckle, P. Piña, A. Medina, A. Miranda, L. Martínez \& J. López. 2013. Combined effect of temperature and food concentration on the filtration and clarification and assimilation efficiency of Atrina tuberculosa sowerby, 1835 (Mollusca: Bivalvia) under laboratory conditions. Archives of Biological Sciences, 65(1), 99-106. http://dx.doi. org/10.2298/ABS1301099N

PRODUCE. 2012. Anuario Estadístico Pesquero y Acuícola 2012. http://www.produce.gob.pe/index.php/estadisticas/anuarios-estadistico> (Acceso 14/10/14).

Rajesh K.V., K.S. Mohamed \& V. Kripa. 2001. Influence of algal cell concentration, salinity and body size on the filtration and ingestion rates of cultivable Indian bivalves. Indian Journal of Marine Science.30: 87-92. <http://cat.inist.fr/?aModele $=$ afficheN\&cpsidt $=14132093>($ Acceso 07/01/15)

Rodhouse P.G. 1978. Energy transformation by the oyster Ostrea edulis (L.) in a temperate estuary. Journal of Experimental Marine Biology and Ecology 34:1-22. http://dx.doi. org/10.1016/0022-0981(78)90053-9. 
Schulte E. 1975. Influence of algal concentration and temperature on the filtration rate of Mytilus edulis. Marine Biology. 30: 331-341. http://dx.doi.org/10.1007/BF00390638

Shumway S.E., T.L. Cucci \& C.M. Yentsch. 1985. Particle selection, ingestion, and absorption in filter-feeding bivalves. Journal of Experimental Marine Biology and Ecology. 91: 77-92. http://dx.doi.org/10.1016/0022-0981(85)90222-9

Tam J., G. Vera, E. Pinto \& G. Sánchez. 2001. Procedimiento Estandarizado de Operación: Método de determinación de la tasa de ingestión de organismos filtradores (PEO-IMP-TI-001). Informe Progresivo Instituto del Mar del Perú. 153: 3-18. < http://biblioimarpe.imarpe.gob.pe:8080/handle/1234 56789/1430> (Acceso 07/01/15)

Velasco L.A. \& J.M. Navarro. 2002. Feeding physiology of infaunal (Mulinia Edulis) and epifaunal (Mytilus chilensis) bivalves under a wide range of concentrations and qualities of seston. Marine Ecology Progress Series 240: 143-155. < http://dspace2.conicyt.cl//handle/10533/ 17645> (Acceso $29 / 01 / 15)$

Ward J.E. \& S.E. Shumway. 2004. Separating the grain from the chaff. Particle selection in suspension and deposit-feeding bivalves. Journal of Experimental Marine Biology and Ecology 300:83-130. http://dx.doi.org/10.1016/j. jembe.2004.03.002

Winter J.E. 1978. A review on the knowledge of suspension-feeding in lamellibranchiate bivalves, with special reference to artificial aquaculture systems. Aquaculture 13: 1-33. http://dx.doi. org/10.1016/0044-8486(78)90124-2
Wolff M. 1985. Abundancia masiva y crecimiento de pre-adultos de la concha de abanico peruana (Argopecten purpuratus) en la zona de Pisco bajo condiciones de "El Niño" 1983. pp. 87-90. En: Arntz, W., A. Landa y J. Tarazona (eds.). "El Niño" y su impacto en la fauna marina. Boletín Instituto del Mar del Perú-Callao, Volumen extraordinario. < http://biblioimarpe.imarpe.gob.pe:8080/handle/123456789/1173> (Acceso 12/12/14)

Wolff M. 1987. Population Dynamics of the Peruvian scallop Argopecten purpuratus during the El Niño Phenomenon of 1983. Canadian Journal of Fisheries and Aquatic Sciences 44 (10): 1684-91. http://dx.doi.org/10.1139/f87-207.

Wolff M. \& R. Wolff. 1983. Observations on the utilization and growth of the pectinid Argopecten purpuratus in the fishing area of Pisco, Perú. Boletín Instituto del Mar del Perú. 7(6): $197-$ 235. < http://biblioimarpe.imarpe.gob.pe:8080/ handle/12 $3456789 / 1038$ ? show=full $>$ (Acceso 29/01/15)

Yamashiro C. \& J. Mendo. 1988. Crecimiento de la concha de abanico (Argopecten purpuratus) en la Bahía Independencia, Pisco, Perú. pp. 163-168. En: Salzwedel H. y A. Landa (eds.) Recursos y dinámica del ecosistema de afloramiento peruano. Boletín Instituto del Mar del Perú- Callao, Volumen extraordinario. < http://biblioimarpe.imarpe.gob. pe:8080/ handle/123456789/1097> (Acceso 14/10/14). 